\title{
Identification of Shear Modulus of Gelatin Blended with Carboxymethylcellulose Scaffolds Using Curve Fitting Method from Compressive Test
}

\author{
Fasai Wiwatwongwana ${ }^{1}$, Yotthana Khunathon ${ }^{1,2}$, Wetchayan Rangsri ${ }^{1}$, Nattawit Promma ${ }^{1} \&$ Somchai Pattana ${ }^{1,2}$ \\ ${ }^{1}$ Department of Mechanical Engineering, Faculty of Engineering, Chiang Mai University, Chiang Mai, Thailand \\ ${ }^{2}$ Biomedical Engineering, Faculty of Engineering, Chiang Mai University, Chiang Mai, Thailand \\ Correspondence: Fasai Wiwatwongwana, Department of Mechanical Engineering, Faculty of Engineering, \\ Chiang Mai University, Chiang Mai 50200, Thailand. Tel: 66-686-830-3949. E-mail: fasaiw227@gmail.com
}

\author{
Received: August 16, 2012 Accepted: August 31, 2012 Online Published: September 27, 2012 \\ doi:10.5539/jmsr.v1n4p106 URL: http://dx.doi.org/10.5539/jmsr.v1n4p106
}

\begin{abstract}
Biopolymer scaffolds which made from various ratios of gelatin blended with carboxymethylcellulose (CMC) were studied. The scaffolds were fabricated to porous structure via freeze drying process and crosslinked to induce conjugation of free amide and carboxyl groups in protein structures by using thermal crosslinking techniques. The mechanical properties of the scaffolds were characterized by experimental procedure. In order to evaluate the modeling, we described the stress-strain behavior of the scaffolds by fitting the data to a neo-Hookean model. Results shown that models which evaluated CMC blended gelatin scaffold in the ratio of 80 and 20 of gelatin and $\mathrm{CMC}$ occurred in the highest average in shear modulus which was $11.46 \mathrm{kPa}$ compared to other blended scaffolds. Gelatin scaffold with 10,30 and $40 \%$ of CMC showed dramatically decreased in the shear modulus which were $2.12,1.73$ and $0.85 \mathrm{kPa}$, respectively compared to pure gelatin scaffold with significant different. These results showed the possibility of using CMC as a low cost material to combine with biopolymers for using in tissue engineering applications.
\end{abstract}

Keywords: gelatin, carboxymethylcellulose, shear modulus, hyperelastic material

\section{Introduction}

Loss of skin in patients can result from multiple causes of disease or injury such as ulcer, burn and trauma. Due to the lost of surface area, skin replacement marketplaces have to produce and develop their wound closure which meet many requirements of native skin for patients (Orgill \& Blanco, 2009). Medical science has thus turned to tissue engineering and materials science and engineering to design proper scaffolds for transplantation and reconstruction of tissues and organs (Ma, 2004). Functionally, the skin has two layers which consist of epidermal and dermal layer. The epidermal layer or epidermis is a regenerative tissue which can recovers its structure completely at the site of the defect while the dermal layer or dermis is a nonregenerative tissue which the wound edges contract and close with simultaneous formation of scar tissue at the defected site (Yannas, 2001). To overcome the nonregenerative tissue in the dermis function, many researchers have developed artificial extracellular matrices or scaffolds for supporting the three-dimensional tissue formation at the wound site (Chong et al., 2007; Gopinath et al., 2004; Hiraoka et al., 2003).

The typical biomaterial used for fabrication of the scaffold is collagen which is a natural polymer that is widely used in many tissue repair and regeneration (Park \& Bronzino, 2003). The basic collagen molecule contains three polypeptide $\alpha$-chains which consist of more than 1000 amino acids of each chain (Piez, 1985). Collagen is an excellent biocompatibility because of low toxicity and poor immunogenic reactions (Timpl, 1984; Linsenmeyer, 1982). However, there are some disadvantages of collagen such as a high cost of preparation of pure type I collagen, more hydrophilicity compared with synthetic polymers and difficult to handling (Friess, 1998). To overcome this, the new fabricated scaffolds should have sufficient strength to provide a good handling and an ability to maintain their stability of the 3D structure and pore size upon the contractile force which cause by the growing cells on the scaffold surface, the compressive and tensile force from surrounding tissues (Park \& Bronzino, 2003).

To overcome the high cost of collagen, we took an interest in using gelatin. Gelatin is denatured from collagen 
and the price of gelatin is cheaper than collagen. Scaffold made from gelatin has shown to be positively interacted with cells which have a research approval of in vitro biocompatibility test of gelatin with fibroblast cells. The cells showed a good affinity and proliferation on the gelatin scaffolds after 14 days of culturing without any signs of biodegradation (Lee, 2005). The second biological material used to blend with gelatin scaffold and can improve a strength of scaffold structure is carboxymethylcellulose (CMC). CMC is a derivative of cellulose by reacted with sodium hydroxide and chloroacetic acid. The properties of CMC are a good in viscosity building, flocculation and a high shear stability. CMC is easily available and very cheap compared to other polysaccharides (Biswal \& Singh, 2004).

The scaffold showed a behavior like a rubber (rubber-like material). It can model in the framework of hyperelasticity. Numerous constitutive equations are available in the literature and have recently been compared and used in many works (Sanabria-DeLong et al., 2008; Guo et al., 2007; Wineman, 2005). The scaffolds typically have nonlinear stress-strain responses due to the elastomeric behavior. However, the identification of material parameters which govern the constitutive equation is difficult. In this research, one homogeneous test is considered to identify constitutive parameter, namely uniaxial compressive test. It consists of performing several homogeneous tests which generate one type of strain state as uniaxial compression. For this purpose, the sample geometry and loadings condition are defined beforehand by numerical investigation. Finally the constitutive is defined using a curve-fitting method.

Therefore, the objectives of this research are to investigate gelatin/CMC scaffold for skin substitute application by identifying the constitutive parameter using a curve-fitting method from the homogeneous test as uniaxial compressive with different ratios of CMC blended with gelatin. We focus on the neo-Hookean (Holzapfel, 2000) which is a simple one of hyperelastic material models for describing scaffold's constitutive behavior.

\section{Method}

\subsection{Materials and Preparation of Gelatin/CMC Scaffolds}

Type A gelatin was purchased from BIO BASIC INC, Canada. It was a reagent grade and derived from pork skin with bloom number of $240-270$ and $\mathrm{pH} 4.5-5.5$ at $25^{\circ} \mathrm{C}$. Its viscosity was $3.5-4.5 \mathrm{cps}$ and moisture less than $12.0 \%$. Carboxymethylcellulose sodium salt (CMC) was purchased from Sigma-Aldrich, St. Louis, MO, USA. It was medium viscosity which was $400-800 \mathrm{cps}$ in a $2 \%$ aqueous solution at $25^{\circ} \mathrm{C}$. We used a deionized water from our laboratory for preparing the gelatin and $\mathrm{CMC}$ solutions.

For the preparation of the gelatin/CMC scaffolds briefly, gelatin powder was immersed in deionized water at room temperature for 0.5 hour before dissolved under agitation for $1 \mathrm{~h}$ at $50^{\circ} \mathrm{C}$ to obtain $0.8 \mathrm{wt} \%(\mathrm{w} / \mathrm{w})$ gelatin solution. Then, $\mathrm{CMC}$ powder was dissolved in deionized water at $70^{\circ} \mathrm{C}$ for 1 hour to form a $0.8 \mathrm{wt} \%(\mathrm{w} / \mathrm{w}) \mathrm{CMC}$ solution. The gelatin solution was blended with the $\mathrm{CMC}$ solution in various ratios as showed in Table 1 . All of blended solutions were stirred for 1 hour at $50^{\circ} \mathrm{C}$ and then degassed on the hotplate. After that it was pipetted into 24-well cell culture plate. The gelatin/CMC solutions were fabricated into porous structure by freeze drying process which freezed at $-20^{\circ} \mathrm{C}$ overnight and lyophilize at $-50^{\circ} \mathrm{C}$ for $24 \mathrm{~h}$. Finally, the scaffolds were dehydrothermal (DHT) treatment by using vacuum oven under condition $140^{\circ} \mathrm{C}$ for $48 \mathrm{~h}$.

Table 1. Blending composition of gelatin/CMC

\begin{tabular}{cc}
\hline \multicolumn{2}{c}{ Blending composition } \\
\hline Gelatin & CMC \\
100 & 0 \\
90 & 10 \\
80 & 20 \\
70 & 30 \\
60 & 40 \\
\hline
\end{tabular}

\subsection{Curve Fitting Method}

The data distribution from stress-strain relations was fit by hyperelastic model which was constitutive law of hyperelastic material using neo-Hookean potential function. NonlinearLeastSquares was used as the alternative 
method. The equation used to determine the parameter $\mathrm{G}$ was shown as follows (Holzapfel, 2000)

$$
T=G\left((\varepsilon+1)-\frac{1}{(\varepsilon+1)^{2}}\right)
$$

Where

$\mathrm{G}$ is initial shear modulus

$T$ is engineering stress

$\varepsilon$ is strain

\subsection{Mechanical Properties of Material}

The nonlinear stress-strain relation of scaffolds typically was known as nonlinear deformation response. The hyperelastic materials have a very small compressibility which is referred to incompressibility. The constitutive behavior of hyperelastic materials are usually derived from the strain energy potentials $(W)$. The strain energy potential function is a scalar function of the strain or deformation tensors which its derivative respects to a strain component that determines the stress component. Their relationship can be written as follows (Holzapfel, 2000)

$$
\sigma_{i}=2\left[\left(\lambda_{i}^{2}-\lambda_{j}^{2}\right) \frac{\partial W}{\partial I_{1}}-\left(\frac{1}{\lambda_{i}^{2}}-\frac{1}{\lambda_{j}^{2}}\right) \frac{\partial W}{\partial I_{2}}\right]
$$

where

$\sigma$ is the Cauchy stress tensor

$W$ is the strain energy function

$\lambda$ is the principle stretch

$I$ is the principle invariant

The neo-Hookean model (Sanabria-DeLong et al., 2008) which its form likes a linear has been used to evaluate various non-linear large deformation of hyperelastic materials. Because the neo-Hookean model is the best for determining the scaffold modulus, it can capture the nonlinear behavior and fit the data, even at larger strains. However, the neo-Hookean model is chosen to be the most appropriate because only one parameter fit is needed, and it can be statistically derived to give the fit parameter a physical meaning. Therefore, we used this model to characterize the nonlinear stress-strain responses of gelatin/CMC scaffold. The specific form of the neo-Hookean constitutive relationship is the strain energy potential function $(W)$ which is depends on the first invariant of the deformation tensor $\left(I_{l}\right)$ by the constant $C_{l}$ which can be written in the following equation.

$$
W=C_{1}\left(I_{1}-3\right)
$$

where

$$
C_{l}=G / 2 \text { and } G \text { is the initial shear modulus of materials }
$$

From the simple extension, the engineering stress in simple extension of an isotropic incompressible hyperelastic material can be written by

$$
T_{11}=2\left(\lambda-\frac{1}{\lambda^{2}}\right)\left(\frac{\partial W}{\partial I_{1}}+\frac{\partial W}{\partial I_{2}}\right)
$$

Where $\lambda$ is equal to the extension ratio which is related to the strain $(\varepsilon)$ by the following expression

$$
\lambda=\varepsilon+1
$$

The invariants $I_{i}$ are given by

$$
I_{1}=\lambda_{1}^{2}+\frac{1}{\lambda_{1}^{2}}+1
$$




$$
\begin{aligned}
& I_{2}=I_{1} \\
& I_{3}=1
\end{aligned}
$$

Therefore the expression of engineering stress $\left(T_{11}\right)$ with the form of neo-Hookean strain energy potential can be written as

$$
T_{11}=G\left(\lambda-\frac{1}{\lambda^{2}}\right)
$$

\subsection{Geometry and Loading Condition}

All of gelatin/CMC scaffolds were approximately $4.7 \mathrm{~mm}$ in height and $13.8 \mathrm{~mm}$ in diameter as shown in Figure 1. For the statistical analysis, all experiments were repeated into five times (the results from five repeated samples showed insignificant different). The compressive modulus and shear modulus of the scaffolds were plotted in the same graph to show the data distribution and significant differences between different ratios of gelatin blended with CMC scaffolds and pure gelatin scaffold. The significant differences between two groups were evaluated using a student t-test with $95 \%$ confidence interval. The differences were considered to be a statistically significant when $\mathrm{p}<0.05$.

To evaluate the homogeneous compressive response of scaffolds, we used a Universal Testing Machine (UTM, Instron No. 5566, USA). The loading condition which applied to the machine was a constant deformation rate of $0.5 \mathrm{~mm} / \mathrm{min}$ in the dry state at $25{ }^{\circ} \mathrm{C}$ (Wang et al., 2007). The values were evaluated from initial compressive stress-strain curve which determine the slope from $10 \%$ to $30 \%$ strain of the scaffolds. The values were expressed as mean \pm standard deviation $(n=5)$ and the raw data of compressive stress-strain of each scaffold was keep to evaluate shear modulus by used the neo-Hookean model. The shear moduli of all different scaffolds were identified by fitting the stress-strain data by a neo-Hookean model which described above. All data points were expressed as mean \pm standard deviation $(\mathrm{n}=5)$. The results of mechanical test from both experiment and model were compared in the same graph which was described in the results and discussion.

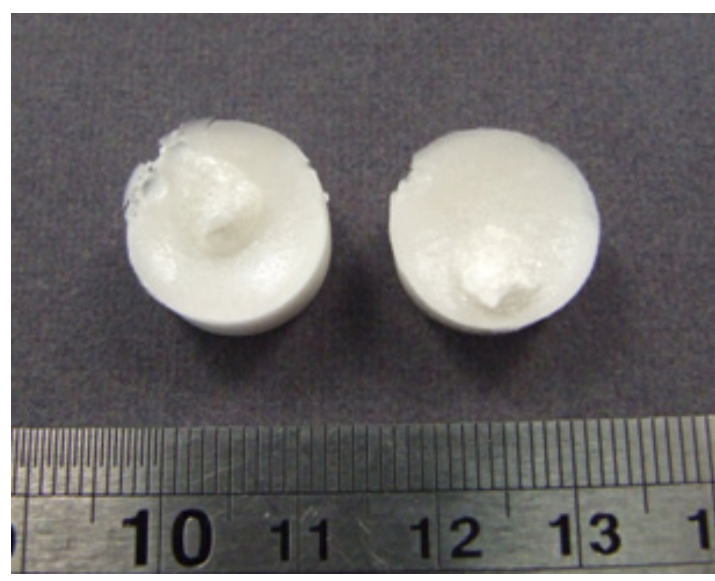

Figure 1. Photograph of $0.8 \%(\mathrm{w} / \mathrm{w})$ gelatin/CMC scaffold which used $30 \%$ of $\mathrm{CMC}$

\section{Results and Discussion}

\subsection{Compressive Modulus of the Scaffolds}

The gelatin/CMC scaffolds were compressed by the UTM with two flat plates to analyzed the mechanical properties of the scaffolds. The example of pure gelatin scaffold compressed by UTM was shown in Figure 2. Force versus displacement was converted into engineering stress and strain by using of the initial dimensions of the scaffolds. 


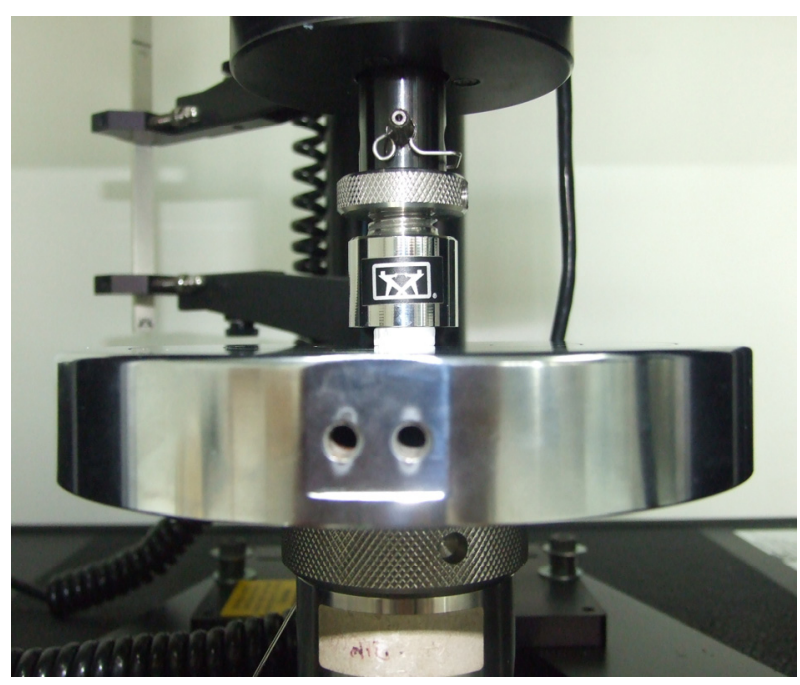

Figure 2. Pure gelatin scaffold ( $0 \%$ CMC) compressed by UTM

The compressive moduli of the scaffolds were plotted in the same graph of shear modulus as shown in Figure 3. All mean values of shear modulus and compressive modulus of all gelatin/CMC scaffolds were represented by dot line and solid line, respectively. The results from compressive modulus showed that gelatin scaffold with $20 \%$ CMC dramatically increased in compressive modulus with significant different compared to pure gelatin scaffold. The compressive modulus of pure gelatin scaffold was $0.21 \pm 0.03 \mathrm{kPa}$ and the compressive modulus of $20 \%$ CMC scaffold was $0.70 \pm 0.07 \mathrm{kPa}$ (Wiwatwongwana \& Pattana, 2011). However, there were some ratios of gelatin/CMC blended scaffold showed decreasing in compressive modulus with significant different compared to pure gelatin scaffold.
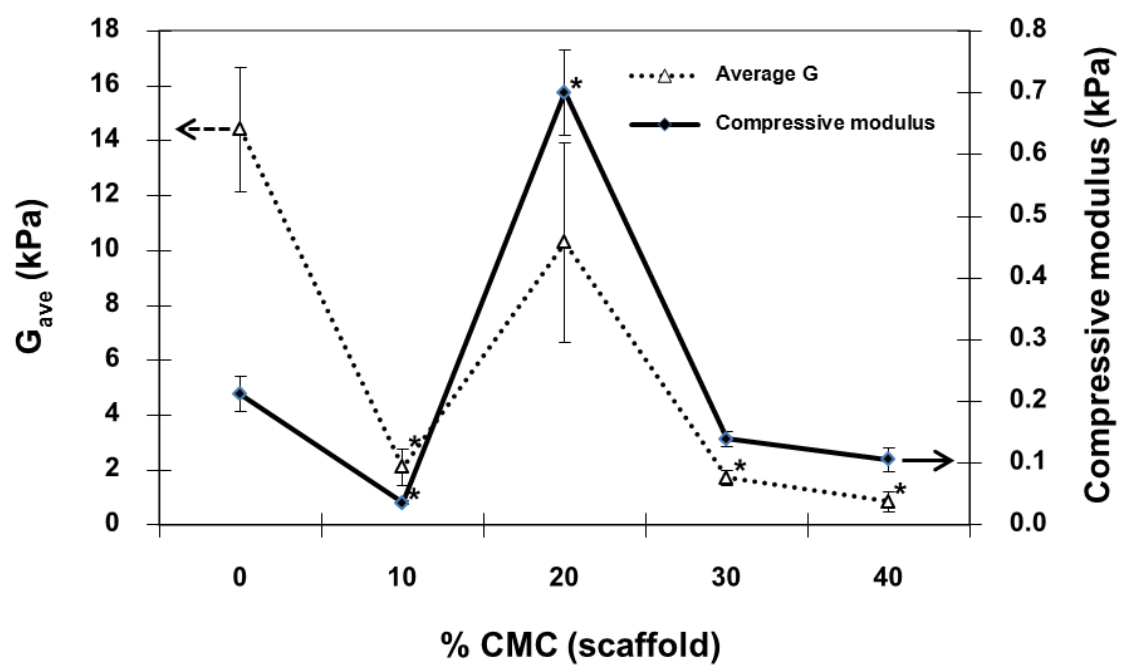

Figure 3. Shear modulus $(\mathrm{kPa})$ in option NonlinearLeastSquares using neo-Hookean model of $4 \%$ strain and compressive modulus $(\mathrm{kPa})$ of $0.8 \%(\mathrm{w} / \mathrm{w})$ gelatin/CMC scaffold $(\mathrm{n}=5)$

( * significant different $p<0.05$ relative to $0 \%$ CMC)

\subsection{Identification of Constitutive Parameter}

The shear modulus of the scaffolds was expressed by fitting data of stress and strain to the neo-Hookean model. We utilized the neo-Hookean constitutive relationship for soft networks which typically express nonlinear behavior of stress-strain curve from compression test (Huang et al., 2005; Lien et al., 2010; Harley et al., 2007). The example of scaffold which fit its stress-strain data to the neo-Hookean model was pure gelatin scaffold $(0 \%$ 
CMC) as shown in Figure 4. Stress curve of the scaffold was nonlinear represented by black points and the neo-Hookean model could fit the curve with $4 \%$ strain represented by orange points. Stress curve of the scaffold was nonlinear represented by (-) and the neo-Hookean model could fit the curve with $4 \%$ strain represented by ( $\square$ ).

All of the scaffolds showed different in shear modulus because it depended on CMC concentration in the gelatin scaffold (Figure 3). Using gelatin blended with $20 \%$ of CMC occurred in the highest value of average shear modulus which was $10.30 \pm 3.64 \mathrm{kPa}$ compared to other ratios of gelatin blended with CMC scaffolds. The shear modulus of the scaffolds decreased with significant different when using $10 \%, 30 \%$ and $40 \%$ of CMC blended with gelatin scaffolds compared to pure gelatin scaffold $(0 \% \mathrm{CMC})$ which was $14.41 \pm 2.24 \mathrm{kPa}$. The shear modulus of gelatin scaffold with $10 \%, 30 \%$ and $40 \%$ of CMC were $2.12 \pm 0.66 \mathrm{kPa}, 1.73 \pm 0.28 \mathrm{kPa}$ and $0.85 \pm$ $0.35 \mathrm{kPa}$, respectively as shown in Table 2 .

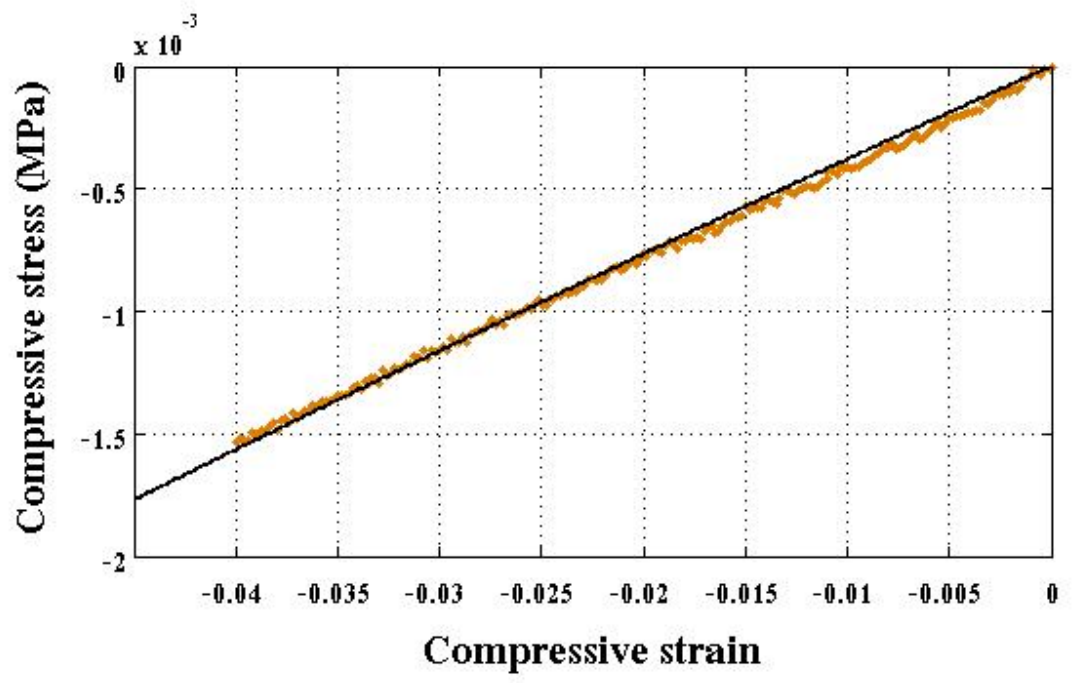

Figure 4. Stress versus strain curve in compression of pure gelatin scaffold $(0 \% \mathrm{CMC})$. Stress curve was nonlinear (-) and fit curve with neo-Hookean model of $4 \%$ strain ( $\square$ )

Table 2. Shear modulus of $0.8 \%(\mathrm{wt} / \mathrm{wt})$ gelatin/CMC scaffold using neo-Hookean model of $4 \%$ strain $(\mathrm{n}=5)$

\begin{tabular}{cccc}
\hline \multirow{2}{*}{ \%CMC (scaffold) } & \multicolumn{2}{c}{ NonlinearLeastSquares } & \\
\hline 0 & Average G $(\mathrm{kPa})$ & Average $\mathrm{RD}$ & $\mathrm{R}^{2}$ \\
10 & 14.408 & 0.00287 & 0.996 \\
20 & 2.117 & 0.00067 & 0.935 \\
30 & 11.463 & 0.00052 & 0.898 \\
40 & 1.730 & 0.00016 & 0.976 \\
\hline
\end{tabular}

\subsection{Comparison of Experimental Test and Neo-Hookean Model}

The results of both compressive modulus from the experimental test and shear modulus from fitting the stress-strain data to the neo-Hookean model were ploted in the same graph (Figure 3). The data distribution of both model (shear modulus) and experiment (compressive modulus) are in the same trend. The scaffold of $20 \%$ CMC showed both shear modulus and compressive modulus in high level. The other concentrations of CMC added into gelatin scaffolds $(10 \%, 30 \%$ and $40 \%$ of $\mathrm{CMC})$ showed decreasing in both shear modulus and compressive modulus compared to pure gelatin scaffold.

There have some advantages of high value in shear modulus and compressive modulus which express the mechanical strength of the scaffold. The high value in shear modulus and compressive modulus can help the scaffold to maintain a 3D porous structure when immersed in media. It is benefit for implanting the scaffold in 
the patient or culturing fibroblast cell in the scaffold. The strength of porous structure can help cell to receive enough nutrients to growth and differentiation.

\section{Conclusion}

The objective in this paper was to present the determination of mechanical properties for incompressible hyperelastic material behavior, namely scaffolds. This material made by blending gelatin and CMC with various ratios. Mechanical response of this material was obtained by uniaxial compression testing. In case of small deformations, the compressive modulus was investigated. The highest value $(0.70 \mathrm{kPa})$ occurred in the scaffold with $20 \%$ of CMC and this value was higher than in case of pure gelatin scaffold $(0.21 \mathrm{kPa})$ with significant difference. The shear modulus of scaffolds was represented in term of an unknown parameter in neo-Hookean model. Fitting stress-strain test data to the model provided directly the shear modulus. The highest of mean of shear modulus $(11.46 \mathrm{kPa})$ occurred in the scaffold with $80 \%$ of gelatin and $20 \%$ of CMC. However, shear modulus of scaffolds with $10 \%, 30 \%$ and $40 \%$ of CMC showed dramatically decreased which were $2.12,1.73$ and $0.85 \mathrm{kPa}$, respectively. At lower $20 \%$ of CMC, the result from previous study showed that this percentage crosslinking between gelatin and CMC was lower than at $20 \%$ of CMC. At higher $20 \%$ of CMC, the pore size distributions of the scaffold were more than $20 \%$ of CMC. In the near future, the identification of parameters governing other type of hyperelastic model, namely Mooney-Rivlin, will be addressed.

\section{Acknowledgements}

This research was performed under support from the Graduate School, Chiang Mai University and Biomedical Engineering Center, Chiang Mai University, Chiang Mai, Thailand.

\section{References}

Biswal, D. R., \& Singh, R. P. (2004). Characterisation of carboxymethyl cellulose and polyacrylamide graft copolymer. Carbohydr. Polym., 57, 379-387. http://dx.doi.org/10.1016/j.carbpol.2004.04.020

Chong, E. J., Phan, T. T., Lim, I. J., Zhang, Y. Z., Bay, B. H., Ramakrishna, S., \& Lim, C. T. (2007). Evaluation of electrospun PCL/gelatin nanofibrous scaffold for wound healing and layered dermal reconstitution. Acta Biomater., 3, 321-330. http://dx.doi.org/10.1016/j.actbio.2007.01.002

Friess, W. (1998). Collagen-biomaterial for drug delivery. Eur. J. Pharm. Biopharm., 45, 113-136. http://dx.doi.org/10.1016/S0939-6411(98)00017-4

Gopinath, D., Rafiuddin, A. M., Gomathi, K., Chitra, K., Sehgal, P. K., \& Jayakumar, R. (2004). Dermal wound healing processes with curcumin incorporated collagen films. Biomater., 25, 1911-1917. http://dx.doi.org/10.1016/S0142-9612(03)00625-2

Guo, Z. Y., Peng, X. Q., \& Moran, B. (2007). Mechanical response of neo-Hookean fiber reinforced incompressible nonlinearly elastic solids. Int. J. Solids Struct., 44, 1949-1969. http://dx.doi.org/10.1016/j.ijsolstr.2006.08.018

Harley, B. A., Leung, J. H., Silva, E. C. C. M., \& Gibson, L. J. (2007). Mechanical characterization of $\begin{array}{lllll}\text { collagen-glycosaminoglycan } & \text { scaffolds. } & \text { Acta } & \text { Biomater. } & 3,\end{array}$ http://dx.doi.org/10.1016/j.actbio.2006.12.009

Hiraoka, Y., Kimura, Y., Ueda, H., \& Tabata, Y. (2003). Fabrication and Biocompatibility of Collagen Sponge Reinforced with Poly (glycolic acid) Fiber. Tissue Engineering, 9, 1101-1112. http://dx.doi.org/10.1089/10763270360728017

Holzapfel, G. A. (2000). Nonlinear Solid Mechanics: A Continuum Approach for Engineering. New York, NY: Wiley.

Huang, Y., Onyeri, S., Siewe, M., Moshfeghian, A., \& Madihally, S. V. (2005). In vitro characterization of

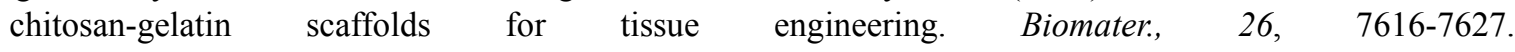
http://dx.doi.org/10.1016/j.biomaterials.2005.05.036

Lee, S. B., Kim, Y. H., Chong, M. S., Hong, S. H., \& Lee, Y. M. (2005). Study of gelatin-containing artificial skin V: fabrication of gelatin scaffolds using a salt-leaching method. Biomater, 26, 1961-1968. http://dx.doi.org/10.1016/j.biomaterials.2004.06.032

Lien, S. M., Ko, L. Y., \& Huang, T. J. (2010). Effect of crosslinking temperature on compression strength of gelatin scaffold for articular cartilage tissue engineering. Mater. Sci. Eng., C, 30, 631-635. http://dx.doi.org/10.1016/j.msec.2010.02.019

Linsenmeyer, T. F. (1982). Immunology of purified collagens and their use in localization of collagen types in 
tissue. In J. B. Weiss, \& M. I. V. Jayson (Eds.), Collagen in Health and Disease (pp. 244-268). Churchill, Living-stone, Edinburgh.

Ma, P. X. (2004). Scaffolds for tissue fabrication. Mater. Today, 30-40. http://dx.doi.org/10.1016/S1369-7021(04)00233-0

Orgill, D., \& Blanco, C. (2009). Biomaterials for treating skin loss. Boca Raton, FL, USA: CRC Press.

Park, J. B., \& Bronzino, J. D. (2003). Biomaterials: Principles and Application. Boca Raton, FL, USA: CRC Press.

Piez, K. A. (1985). Collagen. In J. I. Kroschwitz (Ed.), Encyclopedia of Polymer Science and Engineering (pp. 699-727). New York, NY: Wiley.

Promma, N. (2006). Identification of material parameters using the virtual fields method in case of large deformations. 14-24.

Sanabria-DeLong, N., Crosby, A. J., \& Tew, G. N. (2008). Photo-Cross-Linked PLA-PEO-PLA Hydrogels from Self-Assembled Physical Networks: Mechanical Properties and Influence of Assumed Constitutive Relationships. Biomacromolecules, 9, 2784-2791. http://dx.doi.org/10.1021/bm800557r

Timpl, R. (1984). Immunology of the collagens. In K. A. Piez, \& A. H. Reddi (Eds.), Extracellular Matrix Biochemistry (pp. 159-190). New York, NY: Elsevier.

Wang, M., Xu, L., Hu, H., Zhai, M., Peng, J., Nho, Y., Li, J., \& Wei, G. (2007). Radiation synthesis of PVP/CMC hydrogels as wound dressing. Nucl. Instrum. Methods Phys. Res., Sect. B, 265, 385-389.

Wineman, A. (2005). Some results for generalized neo-Hookean elastic materials. Int. J. Non Linear Mech., 40, 271-279. http://dx.doi.org/10.1016/j.jnonlinmec.2004.05.007

Wiwatwongwana, F., \& Pattana, S. (2011). Modification gelatin scaffold with carboxymethylcellulose for dermal skin. p.1-4. In Proceedings of the 5th International Conference on Bioinformatics and Biomedical Engineering, 10-12 May, 2011. Wuhan, China. http://dx.doi.org/10.1109/icbbe.2011.5780333

Yannas, I. V. (2001). Tissue and organ regeneration in adults. New York, NY: Springer-Verlag. 